\title{
Hybrid closure of common carotid artery perforation caused by misplaced central venous catheter
}

\author{
Konrad Paczkowski ${ }^{1}$, Maciej Chojnicki ${ }^{1}$, Tomasz Sroka ${ }^{1}$, Katarzyna Paczkowska ${ }^{1}$, Katarzyna Gierat-Haponiuk ${ }^{2}$, \\ Ireneusz Haponiuk ${ }^{1,2}$ \\ ${ }^{1}$ Department of Pediatric Cardiac Surgery, St. Adalbertus Hospital, Copernicus, Gdansk, Poland \\ ${ }^{2}$ Chair of Physiotherapy, Faculty of Rehabilitation and Kinesiology, Gdansk Academy of Physical Education and Sport, Gdansk, Poland
}

Adv Interv Cardiol 2018; 14, 4 (54): 440-441

DOI: https://doi.org/10.5114/aic.2018.79878

Mismatched implantation of a venous catheter to the common carotid artery is a rare complication, but unfortunately still occurs. Leaving a catheter in the carotid artery could lead to many life-threatening conditions [1, 2], while catheter removal after time could be dangerous, due to the risk of uncontrolled bleeding [3].

A7-year-old boy with medulloblastoma, after neurosurgical resection of an intracranial tumor, was sent for echocardiography examination, because of unsuccessful administration of the second course of chemotherapy via the central venous catheter (VascuPort), which was implanted 4 weeks earlier. The echocardiography revealed the catheter passing from the right common carotid artery (RCCA) to the aorta with tip of the catheter in the sinus of Valsalva near the right coronary artery. The angioCT scan confirmed the diagnosis and the patient was transferred to the Department of Paediatric Cardiac Surgery in an emergency setting. Because of serious risk of excessive bleeding and planned oncological treatment, the patient was referred for removal of the catheter with simultaneous hybrid closure of the artery perforation.

In the cath lab, the infusion chamber of the VascuPort located in the right axillary line was surgically removed. After slight displacement of the catheter from the ascending aorta to the aortic arch, a guide wire was inserted via the catheter to the descending aorta. The tip of the guide wire was caught with a vascular loop and brought outside the body via a vascular sheath in the right femoral artery. The Amplatzer $4 \mathrm{Fr}$ delivery catheter was inserted over the wire to the aorta. The VascuPort catheter was removed with the simultaneously advanced delivery catheter with kissing technique to avoid bleeding. The Amplatzer Duct Occluder II AS4-2 (ADO II AS; Abbott, MN, USA) was introduced to the RCCA perforation. The device was implanted in the manner to place the proximal disc inside, close to the arterial wall, while the waist and distal disc were located in the canal remaining after the VascuPort catheter. The further course was uncomplicated and the boy continued his oncological treatment using a properly introduced Broviac catheter (Figure 1).

One of the most serious complications of central venous catheterization is arterial puncture. It can lead to arterial occlusion, embolism, pseudoaneurysm formation, dissection of the artery, haemothorax, massive bleeding and compression of the airway tract in consequence. If so, the improperly implanted catheter has to be removed immediately [4]. On the other hand, the perforation of the artery and the tunnel formed after a couple of weeks around the catheter could be difficult to clamp. The perforation could spontaneously reopen, especially in children during chemotherapy. Thus the exact closure of every arterial perforation is so important. Because of the need to preserve growth potential, the use of arterial PTFE coated stents should be avoided in small children. Hybrid strategies that combine surgery with interventional treatment could enable effective treatment in borderline patients.

It is extremely important to confirm the correct position of the catheter directly after implantation in chest $X$-ray and in the case of any doubts perform bedside echocardiography examination.

The ADO II AS device could be used for hybrid closure of arterial perforation, with maintenance of the natural potential for growth in a child.

\section{Conflict of interest}

The authors declare no conflict of interest.

\section{Corresponding author:}

Konrad Paczkowski MD, Department of Pediatric Cardiac Surgery, St. Adalbertus Hospital, Copernicus, 1-6 Nowe Ogrody St, 80-803 Gdansk, Poland, phone: +48 5876848 81, e-mail: konradpaczkowski@gmail.com

Received: 7.05.2018, accepted: 17.07.2018. 

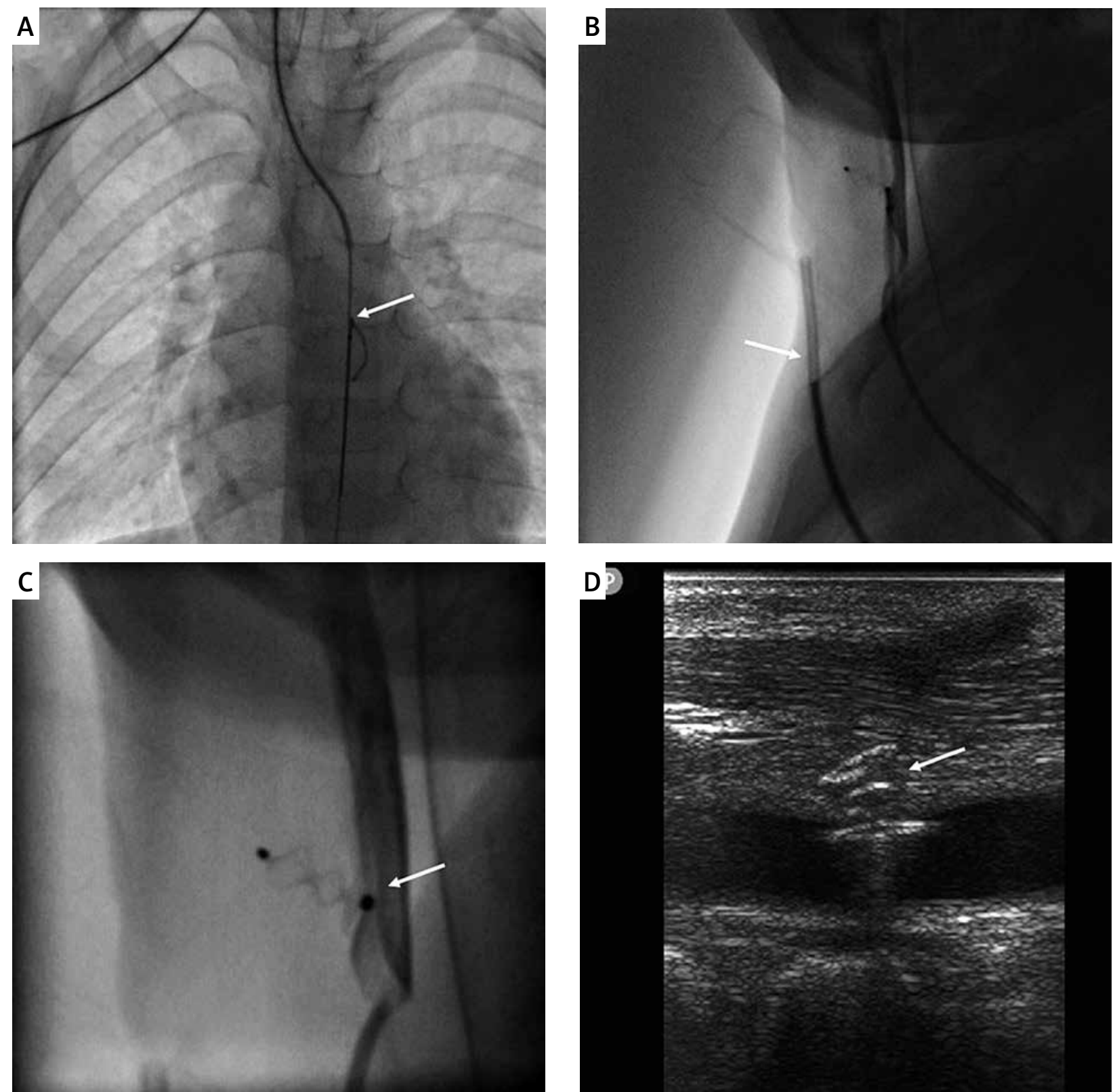

Figure 1. A - The guide wire introduced via the VascuPort catheter was caught with the vascular loop and moved to the descending aorta. The tip of the guide wire was caught with the vascular loop (arrow), B - Amplatzer Duct Occluder II AS introduced to the right common carotid artery (RCCA). VascuPort catheter in subcutaneous tunnel (arrow). C - Control angiography to confirm complete closure of perforation and exclude the presence of arteriovenous fistula. Proximal disc of ADO II AS inside artery - unobstructed flow through the artery (arrow). D - Ultrasonography - proximal disc located close to the artery wall. The waist and distal disc were located in the canal remaining after the VascuPort catheter (arrow)

\section{References}

1. Idialisoa R, Jouffroy R, Saint Martin LC, et al. Transient neurological deficit due to a misplacement of central venous catheter despite ultrasound guidance and ultrasound assistance. Anaesth Crit Care Pain Med 2015; 34: 301-2.

2. Rycaj J, Misiołek H, Stoksik P, et al. Evaluation of thromboembolic complications in children treated for acute lymphoblastic leukemia with Vascuport catheters. Wiad Lek 2005; 58: 41-6.
3. Sarach J, Zschokke I, Melcher GA. A life-threatening mediastinal hematoma after central venous port system implantation. Am J Case Rep 2015; 16: 904-7.

4. Göksel OS, El H, Onalan A, et al. Successful removal of a malpositioned hemodialysis catheter into the aortic arch. J Vasc Access 2012; 13: 543. 